\title{
Efecto de aceites esenciales naturales en el monitoreo de trips del tomate (Frankliniella schultzei Trybom) en Gerbera jamesonii (H. Bolus) (Asteraceae)
}

\section{Effect of natural essential oils in monitoring tomato thrips (Frankliniella schultzei Trybom) in Gerbera jamesonii (H. Bolus) (Asteraceae)}
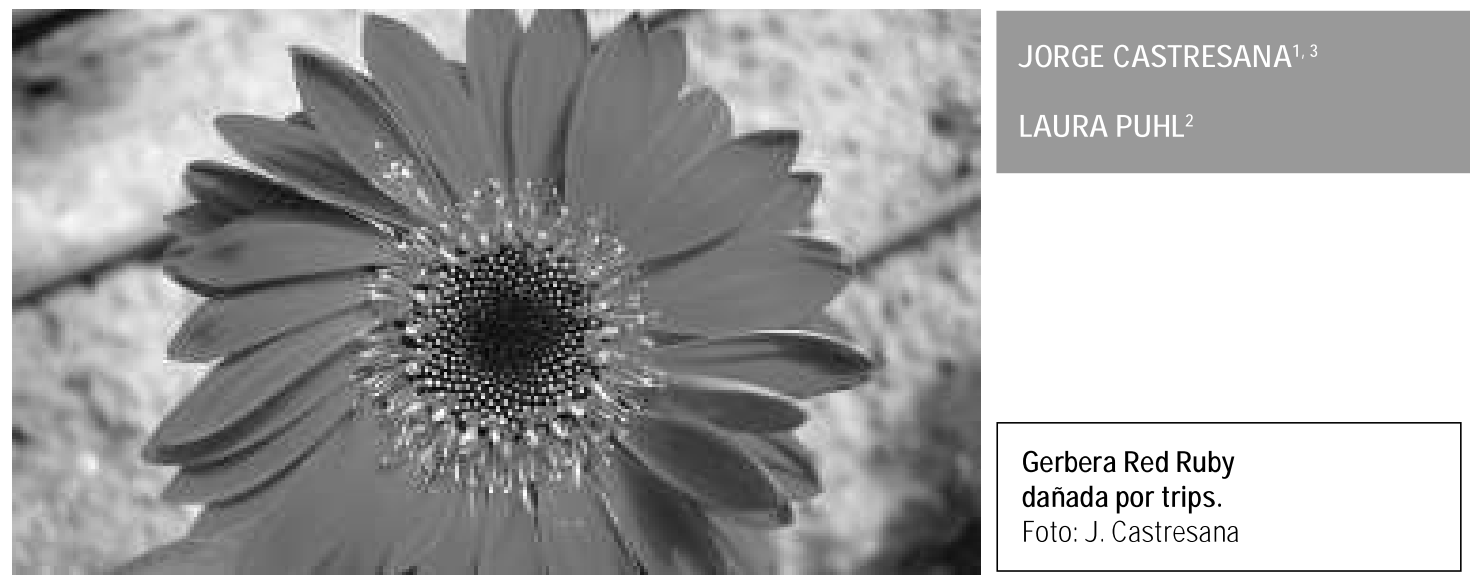

\section{RESUMEN}

El trips Frankliniella schultzei Trybom (Thysanoptera: Thripidae) es una especie polífaga que se alimenta de varias especies ornamentales y hortícolas, y puede causar daños directos e indirectos en cultivos; tanto adultos como ninfas se alimentan de polen y tejido floral. El estudio que se reporta en este artículo se Ilevó a cabo con el objetivo de evaluar el efecto atractivo de aceites esenciales naturales de canela y romero colocados sobre trampas adhesivas de color rojo, empleadas para el monitoreo de trips en gérbera. Se demostró que el promedio de trips adultos capturados fue mayor en las trampas con aceite esencial natural de canela (AC1), seguido por las de aceite esencial natural de romero (AC2) y, por último, el control (sin aceite esencial natural). Estos resultados mostraron que los aceites esenciales naturales colocados en las trampas aumentaron significativamente la captura de trips en trampas adhesivas, en comparación con el control (sin aceite esencial natural).

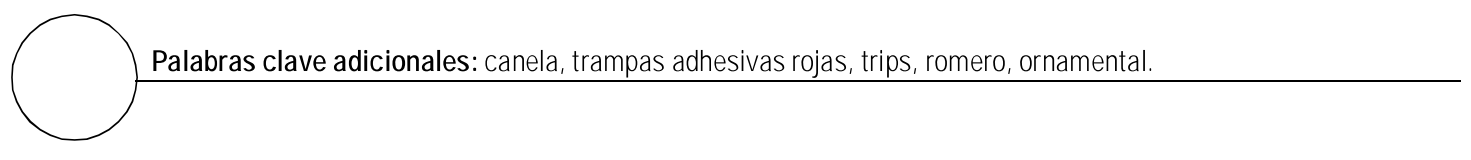

Instituto Nacional de Tecnología Agropecuaria (INTA), EEA Concordia, Estación Yuquerí Concordia, Entre Ríos (Argentina).

2 Facultad de Agronomía, Cátedra de Métodos Cuantitativos Aplicados, Universidad de Buenos Aires, Buenos Aires (Argentina).

3 Autor para correspondencia. jcastresana@correo.inta.gov.ar 


\begin{abstract}
Thrips Frankliniella schultzei (Trybom) (Thysanoptera: Thripidae) is a polyphagous species, feeding on several ornamental plants and horticultural species in different parts of the world, with a feeding behavior that causes both direct and indirect damage to crops. Both adults and nymphs feed on pollen and flower tissue. This present study was carried out in order to know the at traction effect of various natural essential oils such as cinnamon and rosemary added to sticky red traps, which were applied for monitoring thrips in gerbera. It was shown that the highest average number of adult thrips was caught in sticky traps with cinnamon essential oil (AC1), followed by the traps with rosemary essential oil (AC2) and, finally, the control traps (without essential oil). These results showed that essential oils added to traps considerably increased the capture of thrips in sticky traps $(P=0.0316)$ as compared to the control traps (without essential oil).
\end{abstract}

Additional key words: cinnamon, sticky red traps, thrips, rosemary, ornamental.

Fecha de recepción: 30-04-2010

Aprobado para publicación: 02-06-2010

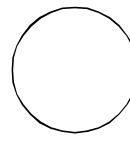

El trips Frankliniella schultzei Trybom (Thysanoptera: Thripidae) es una especie polífaga que se alimenta de varias especies ornamentales y hortícolas, en diferentes partes del mundo (Milne et al., 1996); se asocia a flores de ciertas especies de plantas, incluyendo algunas de importancia económica, de diferentes familias (Johansen, 2002). Los trips adultos y las ninfas causan daños en flores, frutas y hojas; en la flor, la alimentación causa un plateado, veteado, bronceado o la deformación de pétalos y sépalos.

La longitud de onda del espectro visible reflejada por un objeto es uno de los factores que influye en la percepción de los patrones visuales de los insectos, o en cómo estos son atraídos hacia sus plantas hospederas. Así, el número de trips capturados depende de la cantidad relativa de longitud de onda reflejada en la superficie de una trampa, en los niveles de $350 \mathrm{~nm}$ (UV), $440 \mathrm{~nm}$ (azul) y $550 \mathrm{~nm}$ (amarilla) (Vernon y Gillespie, 1990). Las trampas adhesivas de color son adecuadas y eficaces herramientas para el monitoreo de plagas en invernáculos; su acción se basa en el hecho de que ciertas formas voladoras de los insectos son atraídas por ciertos colores, causando su captura con adhesivo entomológico adherido a su superficie (Baranowski y Górski 1991; Górski 1999, 2001). Muchos autores han evaluado la efectividad de trampas pegajosas de colores para estimar poblaciones de F. occidentalis en distintos cultivos, con resultados no siempre coincidentes (Brodsgaard, 1989; Steiner, 1990; Shipp y Zariffa, 1991; Heinz et al., 1992; Higgins, 1992; Higgins y Myers, 1992; Vernon y Gillespie, 1995); las diferencias encontradas por estos y otros autores pueden atribuirse al color de la trampa, pero también podría haber un efecto de la planta hospedera. Yudin et al. (1987) obtuvieron resultados similares usando trampas con azul marino, amarillo y blanco en cultivos de lechuga (Lactuca sativa L.), mientras que Gillespie y Vernon (1990) y Cabello et al. (1991) observaron una preferencia clara de $F$. occidentalis por azul claro, en lugar de blanco y amarillo, en sandía (Citrullus lanatus Thunb.) y pepino de ensalada (Cucumis sativus L.). Yakua et al. (2007) afirman que la captura de F. schultzei con trampas adhesivas de color rojo resultó más efectiva, en comparación con otros colores. Ciertos olores han sido registrados de comportamiento atractivo a plagas de invernadero. Compuestos naturales de la esencia de la flor eugenol y geraniol fueron atractivos para el trips Frankliniella occidentalis (Pergande); la adición de esos compuestos incrementó significativamente la atracción de los adultos hacia las trampas azules en experimentos de laboratorio (Frey et al., 1994). Capturas de trips por medio de diferentes aldehídos aromáticos 
fueron estudiadas por varios investigadores (Brodsgaard, 1990; Teulon y Ramakers, 1990; Teulon et al., 1993).

Estudios Ilevados a cabo por Górski (2001) mostraron un aumento del número de trips de las flores capturados en trampas adhesivas con los aceites esenciales de canela (Cinnamomum zeylanicum), ciprés (Cupressus sempervivens) y romero (Rosmarinus officinalis). Floris et al. (1996) señalan como principales componentes del aceite de la canela el aldehído cinamico (79,3\%), el eugenol $(11,9 \%)$ y el a-pineno $(1,8 \%)$, mientras que Ozcan y Chalchat (2008) determinaron que p-cimeno (44,02\%), linalool $(20,5 \%$, gamma terpenino (16,62\%), timol (1,81\%), b-pineno $(3,61 \%)$, a-pineno $(2,83 \%)$ y eucaliptol $(2,64 \%)$ son los principales componentes del romero. La atracción de las trampas adhesivas puede ser incrementada usando aceites esenciales naturales en combinación con diferentes colores (Frey et al., 1994). El objetivo de este trabajo fue evaluar la atracción de Frankliniella schultzei hacia trampas de color con dos tipos de aceites esenciales naturales, el de canela (Cinnamomum zeylanicum) y el de romero (Rosmarinus officinalis).

\section{MATERIALES Y MÉTODOS}

Los estudios sobre el efecto de aceites naturales en monitoreo de trips (Frankliniella schultzei) fueron llevados a cabo en los meses de enero y febrero de 2009, en el invernáculo de floricultura de la Estación Experimental INTA Concordia, cuya temperatura y humedad relativa fueron $24^{\circ} \mathrm{C}$ y $65 \%$, respectivamente, que favorecen el desarrollo de al tas densidades de poblaciones del trips F. schultzei. Por lo tanto, las poblaciones más altas se observan en las estaciones de verano, con altas temperaturas y alta humedad relativa.

Los aceites esenciales que se estudiaron fueron colocados en trampas adhesivas de color, hechas de cartón Tetra brik y recubiertas con polietileno rojo (color equivalente al generado por el software Photoshop, con un R: 241/251 G: 43/53 B: 11/ 21), con un tamaño de $10 \times 20 \mathrm{~cm}$. Para la confección de las trampas se aplicó el adhesivo para insectos TUTE Hormigas (Envasadora Caseros S.R.L, Carlos Pellegrini 1360, Florida, Prov. Buenos Aires) en ambas caras, dejando solamente sin adhesivo $1 \mathrm{~cm}$ del borde inferior, para el aceite esencial natural, que fue adicionado puro en la cantidad de 0,1 $\mathrm{mL}$ con una periodicidad de dos veces por semana, para evitar la pérdida de atracción por evaporación del aceite esencial en la trampa adhesiva roja. El ensayo fue realizado en 3 canteros ( $1 \mathrm{~m}$ de ancho por $3,5 \mathrm{~m}$ de largo), en cada uno de los cuales se colocaron 20 plantas de gérberas (Gerbera jamesonii var. Testarrosa y var. Ruby Red) en macetas, variedades de flor de color rojo muy atacadas por trips. En estos ensayos se probaron dos aceites esenciales: aceite de hoja de canela y aceite de romero, ambos adquiridos en Euma S.A., Curupayti 1674, Villa Adelina, San Isidro, Prov. Buenos Aires, Argentina. Como testigo se utilizaron trampas adhesivas rojas sin aceites esenciales. Todas las trampas adhesivas rojas se ubicaron suspendidas en forma vertical sobre el tope de las flores de las plantas de gérbera. Se realizó un diseño experimental por bloques completamente aletorizados, donde por cantero se colocaron en combinación, a la misma distancia, tres trampas adhesivas rojas: trampa adhesiva roja sin aceite esencial (control), trampa adhesiva roja con aceite esencial de canela (AC1) y trampa adhesiva roja con aceite esencial de romero (AC2). La ubicación de las trampas dentro de cada cantero fue al azar, y fueron extraídas después de 15 d de exposición para contabilizar el número de trips adultos capturados. Esta operación se repitió tres veces y se promediaron los valores del número de trips capturados. Se realizó un análisis de la varianza para determinar diferencias significativas en el número promedio de trips capturados por las trampas y sus correspondientes aceites. Las comparaciones a posteriori se realizaron con la prueba de Tukey con un nivel de significancia de $5 \%$.

\section{RESULTADOS Y DISCUSIÓN}

Tal como se puede observar en la tabla 1, los aceites esenciales aumentaron significativamente la captura de trips en trampas adhesivas $(P=0,0316)$; el promedio de trips adultos capturados fue mayor en las trampas con $\mathrm{AC} 1$, seguido por AC2 y, por último, el control (figura 1). Por otro lado, no se encontraron diferencias significativas entre el número de insectos 
Tabla 1. Análisis de varianza de la respuesta de F. schultzei en ensayos con aceites esenciales en trampas adhesivas rojas.

\begin{tabular}{|l|r|r|r|r|c|}
\hline \multicolumn{1}{|c|}{ F.V. } & SC & GI & CM & Valor-F & Valor-P \\
\hline Modelo & $1.026,20$ & 4 & 256,56 & 4,76 & 0,0801 \\
\hline Tratamiento & 997,39 & 2 & 498,69 & 9,24 & 0,0316 \\
\hline Parcela & 28,85 & 2 & 14,42 & 0,27 & 0,7781 \\
\hline Error & 215,82 & 4 & 53,95 & & \\
\hline Total & $1.242,06$ & 8 & & & \\
\hline
\end{tabular}

capturados con trampas adhesivas con AC2 y las trampas control (tabla 2). Los resultados obtenidos en el presente trabajo son apoyados por estudios realizados por Górski (2001), quien encontró un incremento estadísticamente significativo en la captura de trips adultos de Frankliniella occidentalis hacia trampas adhesivas de color azul con aceite de canela.

Tabla 2. Prueba de Tukey de comparaciones múltiples entre medias (Alfa $=0,05$; Error $=53,95 ; \mathrm{gl}=4$ ).

\begin{tabular}{|l|c|}
\hline \multicolumn{1}{|c|}{ Tratamiento } & Medias \\
\hline Modelo & $1.026,20$ \\
\hline Control & $36,00 \mathrm{a}$ \\
\hline AC2 & $44,50 \mathrm{ab}$ \\
\hline AC1 & $61,33 \mathrm{~b}$ \\
\hline
\end{tabular}

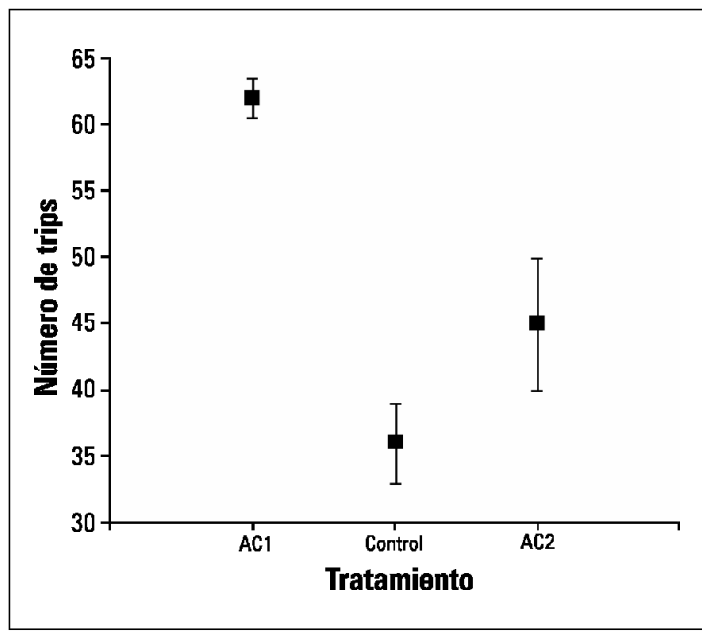

Figura 1. Efecto de los aceites esenciales de canela (AC1) y de romero (AC2) sobre la captura de trips en trampas adhesivas de color rojo.

Promedios con letras distintas indican diferencia significativa $(P \leq 0,05)$.

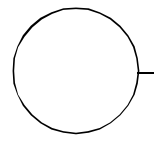

AGRADECIMIENTOS

Se agradece muy especialmente al Ing. Agr. Rubén Larrosa por la identificación de trips para este ensayo, a la Ing. Elena Gagliano por los materiales y plantas de gérbera, a la Dra. Silvina Bado por su cooperación en la redacción y a la traductora pública Mónica Castresana. 


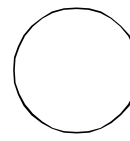

Baranowski, T. y R. Górski. 1991. Przydatnoœæ kolorowych tablic $w$ ochronie roœlin szklarniowych przed szkodnikami. pp. 39-43. En: Materia $^{3}$ y XXXI Sesji Naukowej IOR. Cz. 2, Postery.

Brodsgaard, H.E. 1989. Coloured sticky traps for Frankliniella occidentalis (Pergande) (Thysanoptera: Thripidae) in glasshouses. J. Appl. Entomol. 197, 136-140.

Brodsgaard, J.F. 1990. The effect of anisaldehyde as a scent attractant for Frankliniella occidentalis (Thysanoptera: Thripidae) and the response mechanism involved. SROP/WPRS Bull. 13(5), 3638

Cabello, T.; M.M. Abad y F. Pascual. 1991. Catches of F. occidentalis in traps of distinct colour in protected crops. Boletín de Sanidad Vegetal - Plagas 17, 265 270

Frey, J.E.; R.V. Cortada y H. Helbing. 1994. The potential of flower odours for use in population monitoring of western flower thrips Frankliniella occidentalis Perg. (Thysanoptera: Thripidae). Biocontr. Sci. Technol. 4(2), 177-186.

Floris, I.; C. Carta y M.D. Moretti. 1996. Activites in vitro de plusieurs huiles essentielles sur Bacillus larvae White et essai au rucher. Apidologie 27(2), 111-119.

Gillespie, D.R. y R.S. Vernon.1990. Trap catch of western flower thrips (Thysanoptera: Thripidae) as affected by color and height of sticky traps in mature greenhouse cucumber crops. J. Econ. Entomol. 83, 971-975

Górski, R. 1999. Monitorowanie szkodników roœlin szklarniowych. Progr. Plant Prot./ Post.Ochr. Roœl. 39(1) $321-325$

Górski, R. 2001. Barwne pu³apki chwytne w monitorowaniu szkodników roœlin szklarniowych. Rocz. AR Pozn. Rozpr. Nauk. 310, 3-108

Higgins, C.J. 1992. Western flower thrips in greenhouse populations dynamics, distribution on plants, and association with predators. J. Econ. Entomol. 85,1891-1903

\section{REFERENCIAS BIBLIOGRÁFICAS}

Higgins, C.J. y J.H. Myers. 1992. Sex ratio and population dynamics of western flower thrips (Thysanoptera: Thripidae). Environ. Entomol. 21, 322-330.

Heinz, K.M.; M.P. Parella y J.P. Newman. 1992. Timeefficient use of yellow sticky traps in monitoring insect populations. J. Econ. Entomol. 85, 22632269

Johansen, R.M. 2002. The Mexican Frankliniella fusca (Hinds), F. pallid (Uzel) and F. schultzei (Trybom) species assemblages, in the 'intonsa group' (Insecta, Thysanoptera: Thripidae). Acta Zool. Mex. 85, 5182.

Milne, J.R.; M. Jhumlekhasing y G.H. Walter. 1996 Understanding host plant relationships of polyphagous flower thrips, a case study of Frankliniella schultzei (Trybom). 8-14. En: Goodwin S. y P. Gillespie. (eds). Proc. 1995 Australia and New Zealand Thrips Workshop: Methods, Biology, Ecology and Management, NSW Agriculture. Gosford, Australia.

Ozcan, M.M. y J.C. Chalchat. 2008. Chemical composition and antifungal activity of rosemary (Rosmarinus officinalis L.) oil from Turkey. Int. J. Food Sci. Nutr. 59(7-8), 691-698.

Shipp, J.L. y N. Zariffa. 1991. Spatial patterns of and sampling methods for western flower thrips (Thysanoptera: Thripidae) on greenhouse sweet pepper. Can. Entomol. 123, 989-1000.

Steiner, M.Y. 1990. Determining population characteristics and sampling procedures for the western flower thrips (Thysanoptera: Thripidae) and the predatory mite Amblyseius cucumeris (Acari: Phytoseiidae) on greenhouse cucumber. Environ. Entomol. 19, 1605-1613.

Teulon, D.A.J. y P.M.J. Ramakers. 1990. A review of attractants for trapping thrips with particular reference to glasshouses. IOBC/WPRS Bull. 13, 212-214.

Teulon, D.A.J.; D.R. Penman y P.M.J. Ramakers. 1993. Volatile chemicals for thrips (Thysanoptera: Thripidae) host finding and applications for thrips pest management. J. Econ. Entomol. 86,1405-1415. 
Vernon, R.S. y D.R. Gillespie. 1990. Spectral responsiveness of Frankliniella occidentalis (Thysanoptera: Thripidae) determined by trap catches in greenhouse. Environ. Entomol. 19, 12291241.

Vernon, R.S. y D.R. Gillespie. 1995. Influence of trap shape, and background color on captures of Frankliniella occidentalis (Thysanoptera: Thripidae) in a cucumber greenhouse. J. Econ. Entomol. 88, 288-293.
Yakua, A.; G.H. Walter y A.J. Najar - Rodriguez. 2007 Thrips see red - flower colour and the host relationships of a polyphagous anthophilic thrips. Ecol. Entomol. 32(5), 527-535.

Yudin, L.S.; W.G. Mitchell y J.J. Cho. 1987. Color preference of thrips (Thysanoptera: Thripidae) with reference to aphids (Homoptera: Aphididae) and leafminer in Hawaiian lettuce farms. J. Econ. Entomol. 80, 51-55. 